\title{
Éditorial : Expériences de la santé et du soin en migration : entre inégalités et discriminations
}

Editorial:Unequality or Discrimination? Health and Care Experiences in Migration

Editorial:Experiencias de la salud y la atención médica en el contexto migratorio: entre desigualdades y discriminaciones

Marguerite Cognet, Anne-Cécile Hoyez et Christian Poiret

\section{OpenEdition}

Journals

Édition électronique

URL : https://journals.openedition.org/remi/5861

DOI : 10.4000/remi.5861

ISSN : $1777-5418$

Éditeur

Université de Poitiers

Édition imprimée

Date de publication : 1 juin 2012

Pagination : 7-10

ISBN : 979-10-90426-04-7

ISSN : 0765-0752

Référence électronique

Marguerite Cognet, Anne-Cécile Hoyez et Christian Poiret, «Éditorial : Expériences de la santé et du soin en migration : entre inégalités et discriminations », Revue européenne des migrations

internationales [En ligne], vol. 28 - n² | 2012, mis en ligne le 10 octobre 2012, consulté le 14 avril 2022. URL : http://journals.openedition.org/remi/5861 ; DOI : https://doi.org/10.4000/remi.5861 


\section{Éditorial \\ Expériences de la santé et du soin en migration : entre inégalités et discriminations}

\section{Marguerite COGNET ${ }^{1}$, Anne-Cécile HOYEZ ${ }^{2}$ et Christian POIRET ${ }^{3}$}

a question de la « santé des migrants » est un objet de recherche de longue
date des sciences sociales nord-américaines et plus généralement anglophones. Longtemps ignorée de leurs collègues français, cette thématique fait aujourd'hui une percée importante qui mérite d'être soulignée. L'accroissement des travaux sur ce sujet vient, entre autres, de donner lieu à une première synthèse (Berchet et Jusot, 2012) ainsi qu'à un numéro complet du réputé Bulletin épidémiologique hebdomadaire de l'Institut Nationale de Veille Sanitaire (Lot et Aïna, 2012). Les démographes et économistes de la santé ont été les premiers à publier des analyses statistiques mettant en évidence des écarts significatifs entre les taux de mortalité, les états de santé subjectifs et objectifs des migrants et ceux des individus nés français en France. Par la suite, les travaux de sociologues et d'anthropologues ont apporté des éclairages complémentaires sur l'étude des processus qui conduisent à ces écarts, dont ceux qui sont en partie liés aux inégalités socioéconomiques (Fassin, 2000).

Les données françaises dans le champ de la santé des populations immigrées rejoignent sur nombre de points celles produites par les chercheurs étrangers, notamment quant au poids des déterminants sociaux sur les états de santé. Dans les pays d'immigration, il apparaît nettement que la santé des migrants, d'autant plus bonne à leur arrivée que les politiques migratoires se font sélectives, se détériore au fur et à mesure du temps

1 Enseignante Chercheure, Université Paris Diderot, Laboratoire URMIS (UMR IRD 205), case courrier 7027,75205 Paris cedex 13 ; marguerite.cognet@univ-paris-diderot.fr

2 Chargée de recherche CNRS, Migrinter/Université de Poitiers, 5, rue Théodore Lefebvre, 86000 Poitiers ; anne.cecile.hoyez@univ-poitiers.fr

3 Enseignant Chercheur, Université Paris Diderot, Laboratoire URMIS (UMR IRD 205), case courrier 7027, 75205 Paris cedex 13 ; poiret@univ-paris-diderot.fr 
qu'ils passent sur leur nouveau territoire. Ces constats invitent à regarder de plus près les articulations entre inégalités de santé, inégalités socioéconomiques et discriminations (que celles-ci soient légales ou illégales, directes ou indirectes).

Aujourd'hui, en France comme ailleurs, le consensus se fait parmi les chercheurs sur les liens entre inégalités sociales et inégalités de santé, lesquelles sont « essentiellement le résultat, le produit final des autres inégalités sociales » (Aïach et Fassin, 2004 : 2225). Aux côtés des inégalités socioéconomiques marquant les conditions matérielles de la vie quotidienne (environnement, logement, travail, éducation, etc.), se pose aussi la question des différences dans l'accès aux soins - en termes de droits ou/et d'accessibilité - et dans la qualité de ceux-ci, à raison de la situation migratoire ou/et des origines supposées. Les études françaises les plus récentes en sociologie et anthropologie ont ainsi déplacé leur regard pour interroger l'impact sur le droit à la santé, des ethnicités attribuées aux migrants et à leurs descendants. Le poids des discriminations subies par les minoritaires lors des soins thérapeutiques a ainsi été interrogé (Carde, 2007 ; Cognet et al., 2009) y compris au sein des services hospitaliers (Bertossi et Prud'homme, 2011 ; Cognet et al., 2010). D'autres ont cherché à mieux documenter les pratiques de santé « profanes » parmi les populations migrantes (Guillou, 2009) et les difficultés de recours aux soins de santé primaires qu'elles rencontraient (Hoyez, 2011).

La REMI fait partie des revues qui ont très tôt relayé ces questionnements ${ }^{4}$. Mais ce corpus mérite d'être complété pour prendre en compte l'évolution des politiques publiques, leurs modalités d'application à différentes échelles, leurs impacts sur le recours des migrants aux soins et sur les pratiques de leur prise en charge. Il s'agit donc de prendre en considération l'ensemble des politiques publiques (politiques d'immigration, de santé, sociales) pouvant agir sur l'accès aux soins et susceptibles de peser, en retour, sur les états de santé des migrants et de leurs descendants, ou supposés tels. L'examen de ces questions gagne à être placé dans une perspective comparative entre des pays partageant certains points communs en termes d'origine des populations migrantes, mais aussi en termes de modèles de santé publique et de système de prestations ou, à l'inverse, en permettant de les contraster.

Les articles de cette livraison de la REMI traitent des questions de santé au sens large, en abordant à la fois la question de l'état de santé (déclaré ou perçu) des migrants, celle de l'accès aux soins et des discriminations, ainsi que les pratiques individuelles ou collectives de soins. Les articles de ce numéro analysent les expériences de la santé de populations de migrants - dans différents lieux et à différentes échelles -, à la lumière des contextes sociaux, économiques et culturels. Ils s'articulent autour de deux thèmes majeurs : le premier centre l'analyse sur les effets des inégalités sociales et des processus discriminatoires sur la santé des personnes immigrées, sur leurs possibilités d'accéder aux soins et d'être traitées pour leurs problèmes. Le deuxième s'intéresse davantage aux processus liés à la mobilité des patients, des praticiens et des savoirs de santé, à la

4 La REMI a consacré dix-sept articles à la santé entre 1990 et 2011, dont un dossier en 1990 (vol. 6 $\mathrm{n}^{\circ} 3$ ) et en ouvrant les questions de santé dans différents numéros thématiques (vieillissement, vol. $17 \mathrm{n}^{\circ} 1$; genre, vol. $21 \mathrm{n}^{\circ} 1$; aires régionales, vol. $10 \mathrm{n}^{\circ} 3$, vol. $12 \mathrm{n}^{\circ} 3$, vol. $19 \mathrm{n}^{\circ}$ ) ou numéros de varia (vol. $27 \mathrm{n}^{\circ} 3$ ). 
dynamique des réseaux transnationaux et aux stratégies des migrants comme acteurs contraints par les politiques publiques.

Les quatre premiers articles de ce numéro restituent des résultats d'études centrées sur la production des inégalités de santé. Les auteures s'attachent à démêler l'écheveau des inégalités structurelles socio-économiques et des inégalités dans les états de santé en décryptant les processus complexes qui produisent, entretiennent, mais aussi dissimulent ces inégalités. L'article de Marguerite Cognet et Christelle Hamel, en collaboration avec Muriel Moisy, livre les analyses quantitatives de l'enquête par questionnaire Trajectoires et Origines (TeO) dirigée par l'Institut National des Études Démographiques (INED) qui, pour la première fois en France, permet d'appréhender les différences et les discriminations dans la santé et l'accès aux soins des migrants et de leurs descendants. Les analyses qualitatives de l'enquête par entretiens biographiques (post enquête par questionnaire) éclairent les questions que soulèvent les résultats statistiques quant aux poids des facteurs socioéconomiques, mais également quant à la part de l'origine et du sexe dans la production des inégalités de santé. Les deux articles suivants étudient l'influence que peuvent avoir les représentations des professionnels en charge des personnes malades sur leurs pratiques. Du côté des personnels de l'administration de la santé, Céline Gabarro se penche sur la gestion des dossiers d'AME (Aide Médicale d'État, aujourd'hui réservée aux étrangers en situation irrégulière) dans les Caisses Primaires d'Assurance Maladie (CPAM), dévoilant les enjeux et les pratiques du traitement de ces dossiers au niveau méso-social, partie la plus souvent invisible des processus discriminatoires. L'article de Sylvie Gravel, Jacques Rhéaume et Gabrielle Legendre, basé sur une approche quantitative et qualitative des accidents du travail et des maladies professionnelles au Québec, vient réaffirmer que les inégalités et les discriminations vécues dans le milieu professionnel découlent des inégalités sociales dans leur ensemble et qu'à leur tour elles les renforcent. Les auteurs montrent qu'au Québec comme en France, «l'estimation du droit à être indemnisé » est socialement déterminée (Sayad, 1999 : 271). L'article de Priscille Sauvegrain, quant à lui, présente des données inédites sur les processus de racisation des femmes désignées comme «Africaines » dans les services de maternité. Sur la base d'une longue enquête dans un service de gynéco-obstétrique hospitalier, l'article nous plonge au cœur de la pratique clinique en action. Elle montre que les représentations des cliniciens imprègnent les processus de catégorisation, d'usage courant dans les maternités, et tendent à orienter et à justifier des décisions cliniques qui déterminent les trajectoires de soins pour ces femmes.

Les trois articles suivants engagent la réflexion en partant, cette fois, du point de vue des personnes migrantes malades et des praticiens. Les auteures se concentrent d'une part sur les contraintes que ces personnes perçoivent pour accéder aux soins dans différents pays d'Europe et Amérique du Nord et de l'autre, sur les ressources dont elles disposent et les stratégies qu'elles peuvent mettre en œuvre. Anaik Pian, restitue les résultats d'une enquête qualitative sur les parcours de soins d'étrangers, immigrés en France, et traité pour un cancer. Elle s'intéresse plus particulièrement aux configurations d'entrée et de circulation dans l'espace du soin, mais aussi aux « trajectoires du mourir » de cette population en situation socio-économique et juridique précaire, dans trois villes bretonnes. De son côté, l'article d'Émilie Adam-Vezina retrace les trajectoires d'Africaines de la région des Grands Lacs (en particulier du Burundi et du Rwanda) devenues séropositives 
$\mathrm{au} \mathrm{VIH}+$ dans un contexte de violences. Elle analyse les raisons et les conditions dans lesquelles ces femmes se décident à prendre la route de l'exil. L'auteure revient avec elles sur leur choix du Canada comme pays de « salut», les difficultés qu'elles rencontrent pour obtenir un titre de séjour puis accéder aux soins sur le territoire canadien. Son analyse dévoile les enjeux qui entourent la question de la reconnaissance, une question cruciale au cœur des parcours de migration, de mise en situation de minoritaires, de maladie et de souffrance. Enfin, dans son article, Anne-Cécile Hoyez sort du cadre biomédical pour aborder la question des pratiques de soin émanant d'autres grands systèmes de représentation de la santé et de la maladie. Elle interroge les liens entre présence d'une médecine d'origine indienne et sous-représentativité des migrants de cette région dans ses dynamiques actuelles en analysant le phénomène au prisme de l'expérience migratoire et des dynamiques locales et transnationales des pratiques de santé.

\section{Références bibliographiques}

AÏACH Pierre et FASSIN Didier (2004) L'origine et les fondements des inégalités sociales de santé, Revue du Praticien, 54, pp. 2221-2227.

BERCHET Caroline et JUSOT Florence (2012) État de santé et recours aux soins des immigrés : une synthèse des travaux français, Questions d'économie de la santé, 172, pp. 1-8.

BERTOSSI Christophe et PRUD'HOMME Dorothée (2011) La «diversité » à l'hôpital : identités sociales et discriminations (Synthèse du rapport de l'IFRI sur les discriminations en établissements hospitaliers), Paris, Centre Migrations et Citoyennetés de l'IFRI, 11 p.

CARDE Estelle (2007) Les discriminations selon l'origine dans l'accès aux soins, Santé Publique, 2, pp. 99-109.

COGNET Marguerite, ADAM-VEZINA Émilie and BASCOUGNANO Sandra (2010) Discrimination in Health Care Services, in Zhenyi Li and Thomas Lawrence Long Eds., The Meaning Management Challenge. Making Sense of Health, Illness and Disease, Oxford, United Kingdom, Inter-Disciplinary Press, pp. 3-10.

COGNET Marguerite, GABARRO Céline et ADAM-VEZINA Émilie (2009) Entre droit aux soins et qualité des soins, Hommes et Migrations, 1282, pp. 54-65.

FASSIN Didier (2000) Qualifier les inégalités, in Annette Lederc, Didier Fassin et Hélène Grandjean Éds., Les inégalités sociales de santé, Paris, La Découverte, pp. 123-144.

GUILLOU Anne Yvonne (2009) Immigration thérapeutique, immigration pathogène. Abandonner le « parcours thérapeutique » pour «l'expérience migratoire », Sciences sociales et santé, 27 (1), pp. 63-72.

HOYEZ Anne-Cécile (2011) L'accès aux soins des migrants en France et la « culture de l'initiative locale ». Une analyse des contextes locaux à l'épreuve des orientations nationales, Cybergeo: European Journal of Geography, [en ligne]. URL : http://cybergeo.revues.org/24796

LOT Florence et AÏNA Élodie (Coord.) (2012) Santé et recours aux soins des migrants en France, Bulletin épidémiologique hebdomadaire, 17 janvier 2012 / $\mathrm{n}^{\circ}$ 2-3-4, $40 \mathrm{p}$.

SAYAD Abdelmalek (1999) La maladie, la souffrance et le corps, in Abdelmalek Sayad, La double absence. Des illusions de l'émigré aux souffrances de l'immigré, Paris, Seuil, pp. 255-303. 